\title{
METODOLOGIA PRÁTICA PARA ESTIMAÇÃO DE PARÂMETROS DE GERADORES SÍNCRONOS A PARTIR DE MEDIDAS DE PERTURBAÇÕES
}

\author{
Elmer P. T. Cari* \\ elmer@utfpr.edu.br; \\ Luís F. C. Alberto* \\ lfcalberto@usp.br; \\ Newton G. Bretas ${ }^{\dagger}$ \\ ngbretas@sc.usp.br \\ *Universidade Tecnológica Federal de Paraná - UTFPR Cornélio Procópio, PR, Brasil \\ ${ }^{\dagger}$ Universidade de São Paulo-EESC, São Carlos, SP, Brasil
}

\begin{abstract}
A new practical and robust methodology to estimate parameters of synchronous generators is proposed in this paper. The proposed methodology is based on trajectory sensitivity analysis combined with a new approach termed 'minimization approach' to estimate parameters of nonlinear dynamical systems modeled by a set of differential-algebraic equations. A suitable choice of inputs and outputs allows the division of the estimation of generator parameters into the independent estimation of mechanical and electrical parameters. The methodology is robust with respect to initial parameter guesses and it does not require special tests, but uses disturbance data (3- $\phi$ currents and voltages, field voltage and mechanical rotor speed measurements) collected from the electric power system without disconnecting the generator from the grid.
\end{abstract}

KEYWORDS: Parameter estimation, synchronous generator, trajectory sensitivity.

\section{RESUMO}

Este artigo propõe uma nova metodologia prática e robusta para estimar parâmetros de geradores síncronos. A metodologia proposta utiliza a análise de sensibilidade de trajetória combinada com uma nova abordagem, denominada 'abordagem de minimização' para estimação de parâmetros de sistemas dinâmicos não-lineares modelados por conjuntos de equações algébricos diferenciais. Uma escolha adequada de entradas e saídas permite a divisão da estimação de parâmetros do gerador na estimação independente dos parâmetros elétricos e mecânicos. A metodologia é robusta com relação às condições iniciais dos parâmetros, não requer execução de testes especiais e utiliza apenas medidas de perturbações de fácil obtenção (correntes e tensões trifásicas, tensão de campo e velocidade do rotor) coletadas do sistema de energia elétrica sem desconectar o gerador da rede.

PALAVRAS-CHAVE: Estimação de parâmetros, gerador síncrono, sensibilidade de trajetória. 


\section{Nomenclatura}

\begin{tabular}{|c|c|}
\hline$I_{q}$ & $\begin{array}{l}\text { Correntes de eixo direto e de eixo } \\
\text { em quadratura. }\end{array}$ \\
\hline$I, V_{t}$ & Tensão e corrente terminal do estator \\
\hline$E_{d}^{\prime}, E_{q}^{\prime}$ & $\begin{array}{l}\text { Tensões transitórias de eixo } \\
\text { direto e de eixo em quadratura. }\end{array}$ \\
\hline$P_{e}, Q_{e}$ & Potência ativa e reativa do gerador. \\
\hline & Ângulo de fase da tensão terminal. \\
\hline$\delta$ & $\begin{array}{l}\text { Ângulo de fase da tensão transitória } \\
\text { de eixo em quadratura }\end{array}$ \\
\hline$\beta$ & Ângulo de carga. \\
\hline$x_{d}, x_{q}$ & $\begin{array}{l}\text { Reatâncias de eixo direto e de eixo } \\
\text { em quadratura. }\end{array}$ \\
\hline$r^{\prime}$ & $\begin{array}{l}\text { Reatâncias transitórias de eixo } \\
\text { direto e de eixo em quadratura. }\end{array}$ \\
\hline$T_{d o}^{\prime}, T_{q o}^{\prime}$ & $\begin{array}{l}\text { Constantes de tempo de eixo direto } \\
\text { e de eixo em quadratura. }\end{array}$ \\
\hline$H, D$ & $\begin{array}{l}\text { Constante de inércia e constante de } \\
\text { amortecimento. }\end{array}$ \\
\hline$\lambda_{L}$ & Multiplicador de Lagrange \\
\hline
\end{tabular}

\section{INTRODUÇÃO}

As simulações computacionais são amplamente utilizadas pelos engenheiros para prever o comportamento dinâmico do sistema de energia elétrica (SEE). A partir dos resultados destas simulações, decisões, tais como a determinação dos limites de operação, o ajuste dos esquemas de proteção e o despacho de carga, são tomadas. O sucesso destas decisões depende, em grande parte, da exatidão das simulações computacionais. Portanto, os parâmetros do modelo matemático devem ser muito bem ajustados para garantir que os resultados numéricos destas simulações fiquem suficientemente próximos ao comportamento do sistema real.

Entretanto, os parâmetros estão sujeitos a incertezas devido a diversos motivos, tais como: perda do banco de dados, alteração pelo envelhecimento do equipamento, variação devido ao efeito da saturação, entre outros. Para evitar ou pelo menos minimizar estas incertezas, os engenheiros estimam os parâmetros a partir de medidas obtidas diretamente do sistema em funcionamento.

$\mathrm{Na}$ literatura, existem diversas metodologias para estimação de parâmetros de geradores síncronos, porém só algumas delas estimam os parâmetros usando medições tomadas com o gerador ligado ao sistema. Os ensaios de rejeição de carga (IEEE, 2002), por exemplo, são utilizados para estimar os parâmetros de eixo direto e de eixo em quadratura. Entretanto, tal abordagem requer que o gerador opere em condições especiais e seja retirado do sistema durante o teste para a obtenção das medidas.
Perturbações injetadas intencionalmente na máquina síncrona, como por exemplo, as perturbações PRBS (Pseudo Random Binary Sequence) (Karrari and Malik, 2004), (Vermeulen et al., 2002), (Burth et al., 1999), possibilitam a estimação dos parâmetros a partir de medidas obtidas com o gerador ligado ao sistema. A única dificuldade na aplicação desta metodologia em geradores reais é que se o sinal injetado não for devidamente projetado, a perturbação pode não ser suficiente para excitar a dinâmica do sistema ou, no pior caso, a perturbação pode gerar instabilidade no sistema. Além disso, é necessário a permissão do operador do SEE para a realização deste ensaio.

Outra característica das metodologias existentes para estimar parâmetros de geradores é que o sucesso da correta estimação dos parâmetros depende da disponibilidade de valores iniciais dos parâmetros próximos aos valores verdadeiros. Na literatura, tem-se dado pouca importância a este problema. Em (Sanchez et al., 1988) por exemplo, foram mencionados alguns problemas de convergência da metodologia de sensibilidade de trajetória devido a indisponibilidade de valores iniciais próximos aos valores verdadeiros.

Com o intuito de resolver os problemas mencionados anteriormente propõe-se, neste artigo, uma nova metodologia para estimar parâmetros de geradores síncronos. A proposta que combina a metodologia de sensibilidade de trajetória, como algoritmo de ajuste de parâmetros, com uma nova formulação do problema de estimação de parâmetros denominada 'abordagem de minimização'. A metodologia proposta possui as seguintes características: (i) não requer testes especiais, pois utiliza medidas de perturbações obtidas com a máquina em operação;(ii) estima os parâmetros mecânicos e elétricos de forma desacoplada; (iii) estima o ângulo de rotor como resultado do processo de estimação; (iv) é robusto em relação a valores iniciais de parâmetros ${ }^{1}$, permitindo a convergência do processo de estimação mesmo para valores iniciais de parâmetros distantes dos valores verdadeiros.

O sucesso da metodologia proposta está na concepção de uma nova formulação do problema de estimação de parâmetros. Nesta nova formulação, as equações algébricodiferenciais que modelam o gerador são substituídas por um problema de minimização restrito às equações diferenciais. Esta nova abordagem é denominada neste texto de 'abordagem de minimização', sendo ela, por si só, uma das contribuições originais deste trabalho.

\footnotetext{
${ }^{1}$ Para que um algoritmo de ajuste de parâmetros seja robusto em relação a valores iniciais de parâmetros, ele deve ter uma região de convergência grande, ou seja, deve permitir a correta estimação mesmo que o processo comece com valores iniciais dos parâmetros distantes dos valores verdadeiros.
} 
Este artigo está organizado da seguinte forma: na Seção 2, apresenta-se o algoritmo convencional de estimação de parâmetros de sistemas dinâmicos não lineares via análise de sensibilidade de trajetórias. Na Seção 3, uma nova proposta para estimação de parâmetros de sistemas dinâmicos não lineares modelados por equações algébrico-diferenciais é desenvolvida. A modelagem do problema de estimação de parâmetros do gerador é apresentada na Seção 4. Na Seção 5, apresentam-se os resultados dos testes e, na Seção 6, as conclusões deste trabalho são enumeradas.

\section{SENSIBILIDADE DE TRAJETÓRIA PARA ESTIMAÇÃO DE PARÂME- TROS DE SISTEMAS DINÂMICOS NÃO-LINEARES}

A metodologia de sensibilidade de trajetória é muito utilizada para a estimação de parâmetros de sistemas dinâmicos não lineares. Aplicações desta metodologia podem ser encontradas por exemplo em (Hiskens and Koeman, 1998) e (Benchluch and Chow, 1993). A metodologia de sensibilidade de trajetória possui algumas vantagens quando comparada a outras técnicas de estimação de parâmetros: (i) pode ser facilmente implementada para uma grande classe de sistemas não-lineares, incluindo sistemas com não-linearidades não-suaves difíceis de serem modeladas, como por exemplo, limites de controladores e chaveamentos (Hiskens, 2000), (ii) permite a estimação de parâmetros a partir de janelas amostrais de tempo relativamente curtas e (iii) permite a estimação das condições iniciais das variáveis de estado.

Considere um sistema não-linear modelado por

$$
\begin{aligned}
\dot{x} & =f(x, z, p, u), \\
0 & =g(x, z, p, u), \\
y & =h(x, z, p, u),
\end{aligned}
$$

onde $x \epsilon R^{m}$ é o vetor de estados, $z \epsilon R^{q}$ é o vetor de variáveis algébricas, $y \in R^{r}$ é o vetor de saída, $u \in R^{l}$ é o vetor de entrada e $p \epsilon R^{p}$ é o vetor de parâmetros. Seja $p_{i}$ a $i$-ésima componente do vetor de parâmetros ' $p$ '. Admitese que as funções $f, g$ e $h$ sejam diferenciáveis em relação a cada $p_{i}, i=1, \ldots, p$. As sensibilidades de trajetória $\frac{\partial x(t)}{\partial p_{i}}$ e $\frac{\partial y(t)}{\partial p_{i}}$ das soluções $x(t)$ e da saída $y(t)$ em relação ao parâmetro $p_{i}$ são obtidas derivando-se (1) em relação a $p_{i}$, isto é:

$$
\begin{aligned}
\frac{d}{d t} \frac{\partial x}{\partial p_{i}}= & \frac{\partial f(x, z, p, u)}{\partial x} \cdot \frac{\partial x}{\partial p_{i}}+\frac{\partial f(x, z, p, u)}{\partial z} \cdot \frac{\partial z}{\partial p_{i}}+ \\
& \frac{\partial f(x, z, p, u)}{\partial p_{i}} \\
0= & \frac{\partial g(x, z, p, u)}{\partial x} \cdot \frac{\partial x(t)}{\partial p_{i}}+\frac{\partial g(x, z, p, u)}{\partial z} \cdot \frac{\partial z(t)}{\partial p_{i}}+ \\
& \frac{\partial g(x, z, p, u)}{\partial p_{i}} \\
\frac{\partial y}{\partial p_{i}}= & \frac{\partial h(x, z, p, u)}{\partial x} \cdot \frac{\partial x}{\partial p_{i}}+\frac{\partial h(x, z, p, u)}{\partial z} \cdot \frac{\partial z}{\partial p_{i}}+ \\
& \frac{\partial h(x, z, p, u)}{\partial p_{i}}
\end{aligned}
$$

As funções de sensibilidade de trajetória quantificam a variação da trajetória (solução) em relação à variação de parâmetros. Esta quantificação é utilizada para ajustar o vetor de parâmetros $p$ a fim de minimizar a diferença entre a saída do sistema real e a saída do sistema auxiliar modelo matemático. Mais precisamente, minimiza-se o funcional $J(p)$ :

$$
J(p)=\frac{1}{2} \int_{0}^{T_{o}}\left(y_{m e d}-y\right)^{T}\left(y_{m e d}-y\right) d t
$$

onde, $y_{m e d}$ é o vetor de saída do sistema real (valor medido) e $y$ é o vetor de saída do modelo (1). A condição de otimalidade $\frac{\partial J(p)}{\partial p}=0$ é dada por:

$$
G(p):=\frac{\partial J(p)}{\partial p}=-\int_{0}^{T_{o}}\left(\frac{\partial y}{\partial p}\right)^{T}\left(y_{m e d}-y\right) d t=0 .
$$

O método de Newton pode ser usado para resolver a equação não linear (4). Partindo do vetor inicial $p=p_{o}$, o ajuste dos parâmetros na $k$-ésima iteração é dado por:

$$
p^{(k+1)}=p^{(k)}-\left.h_{o p t} \Gamma(p)^{-1} G(p)\right|_{p=p^{(k)}},
$$

onde $h_{\text {opt }}$ é um passo ótimo, o qual pode ser calculado por meio do método de busca quadrática (Luenberger, 1972), e $\Gamma(p)$ é a matriz obtida derivando-se $G(p)$ em relação ao vetor de parâmetros $p$ (Jacobiana de $\mathrm{G}(\mathrm{p})$ )). A matriz $\Gamma(p)$ pode ser aproximada desprezando-se os termos de segunda ordem:

$$
\left.\Gamma(p) \approx \int_{0}^{T_{o}}\left(\frac{\partial y}{\partial p}\right)^{T}\left(\frac{\partial y}{\partial p}\right) d t\right|_{p=p^{(k)}}
$$


onde $\frac{\partial y}{\partial p}$ é a matriz de sensibilidade. A i-ésima coluna de $\frac{\partial y}{\partial p}$ representa a sensibilidade das variáveis de saídas em relação ao parâmetro $p_{i}$.

A principal desvantagem da metodologia de estimação de parâmetros via sensibilidade de trajetórias é a falta de robustez com relação às condições iniciais dos parâmetros. A região de convergência desta metodologia pode ser inaceitavelmente pequena levando o processo iterativo de estimação a soluções não desejadas e/ou sem sentido físico ou ainda à não convergência. Estes problemas ocorrem devido a dois fatores principais. O primeiro está relacionado a problemas de condicionamento numérico e o segundo a não solvabilidade da equação algébrica $0=g(x, z, p, u)$ do sistema (1) ao longo do processo iterativo de ajuste dos parâmetros.

Os problemas numéricos podem ser minimizados com um procedimento de estimação dos parâmetros em duas fases que será descrito na subseção 2.1. Para resolver os problemas de não convergência devido a não solvabilidade da equação algébrica $0=g(x, z, p, u)$, propõe-se neste artigo uma nova abordagem, denominada abordagem de minimização, que será descrita em detalhes na seção 3 .

\subsection{Estimação em duas fases}

Uma causa frequente de não convergência da metodologia de sensibilidade de trajetória é a existência de parâmetros com diferentes níveis de sensibilidade (ou diferentes níveis de influência na saída). Geralmente os parâmetros com nível de sensibilidade baixa tornam-se críticos no momento do ajuste fazendo o processo divergir. Para contornar este problema, os parâmetros podem ser divididos em dois grupos, um grupo com parâmetros com nível de sensibilidade alto e outro com nível de sensibilidade baixo. Esta divisão pode ser realizada via decomposição QR da matriz jacobiana $\Gamma(p)$ conforme proposto em (Burth et al., 1999). Na primeira fase, os parâmetros com alta sensibilidade são ajustados pelo algoritmo de estimação durante algumas poucas iterações, mantendo-se fixos os parâmetros com baixa sensibilidade. Na segunda fase, todos os parâmetros são ajustados simultaneamente. Esta abordagem aumenta, em geral, a região de convergência do algoritmo de ajuste baseado em sensibilidade de trajetória.

\section{UMA ABORDAGEM DE MINIMIZA- ÇÃO PARA A ESTIMAÇÃO DE PARÂ- METROS DE SISTEMAS DE EQUA- ÇÕES ALGÉBRICO-DIFERENCIAIS.}

Nesta seção, uma nova formulação, denominada abordagem de minimização, do problema de estimação de parâmetros via sensibilidade de trajetória é apresentada. Esta abordagem resolve os problemas de convergência do processo iterativo de estimação de parâmetros decorrentes da não solvabilidade da equação algébrica $0=g(x, z, p, u)$ do sistema (1). Para isto, substitui-se o sistema de equações algébrico-diferenciais (1) por um problema de minimização restrito à equação diferencial. O objetivo desta substituição é relaxar a equação algébrica durante o processo de estimação para evitar os problemas de inexistência da solução da mesma. Matematicamente (1) é substituído por:

$$
\begin{aligned}
& \min _{(x, z)} \sum_{i} g_{i}^{2}(x, z, p, u) \\
& \text { s.a. } \quad\{\quad \dot{x}=f(x, z, u, p)\}, \\
& y=h(x, z, p, u),
\end{aligned}
$$

onde $g_{i}$ representa a $i$-ésima equação algébrica do modelo (1), a equação diferencial é uma restrição do problema de minimização e $y$ é a saída.

O mínimo global esperado para este problema de minimização é zero. Quando o mínimo global é alcançado, $g_{i}=0$ para todo $i$, a solução do problema de minimização (7) coincide com a solução do sistema de equações algébrico-diferenciais (1). Após a convergência de $p$, verifica-se se o mínimo global foi alcançado para validar a estimação dos parâmetros.

A forma discreta de (7) pode ser obtida usando um método de integração implícito tal como a regra trapezoidal, isto é:

$$
\begin{aligned}
& \min _{x_{n+1}, z_{n+1}} \quad \sum_{i} g_{i}^{2}\left(x_{n+1}, z_{n+1}, p, u_{n+1}\right) \\
& \text { s.a }\left\{x_{n+1}-x_{n}-\frac{\Delta t}{2}\left[f\left(x_{n}, z_{n}, p, u_{n}\right)+\right.\right. \\
& \left.\left.f\left(x_{n+1}, z_{n+1}, p, u_{n+1}\right)\right]=0\right\} \text {, } \\
& y_{n+1}=h\left(x_{n+1}, z_{n+1}, p, u_{n+1}\right) \text {. }
\end{aligned}
$$

O método de multiplicadores de Lagrange (Bazaraa, 1979) pode ser usado para resolver o problema de minimização restrito (8). A função Lagrangiana não restrita, 
a qual depende das variáveis $\left(x_{n+1}, z_{n+1}\right)$ e do multiplicador de Lagrange $\left(\lambda_{L(n+1)}\right)$, é dada por:

$$
\begin{aligned}
L= & g^{2}(x, z, p, u)_{n+1}+\lambda_{L n+1}\left[x_{n+1}-x_{n}+\right. \\
& \left.-\frac{\Delta t}{2}\left(f\left(x_{n}, z_{n}, p, u_{n}\right)+f\left(x_{n+1}, z_{n+1}, p, u_{n+1}\right)\right)\right] .
\end{aligned}
$$

As condições de otimalidade de KKT (Karush-KuhnTucker) (Bazaraa, 1979) são: h. Ajuste os parâmetros usando a equação (5) e vá para o passo c.

\section{METODOLOGIA DE ESTIMAÇÃO DE PARÂMETROS DE GERADORES SÍN- CRONOS}

Nesta seção, uma nova metodologia para estimar parâmetros de geradores síncronos é desenvolvida. Explorando a abordagem de minimização, proposta na seção

$\frac{\partial L}{\partial x_{n+1}}=2 g_{n+1} \frac{\partial g_{n+1}}{\partial x_{n+1}}+\lambda_{L(n+1)}\left(1-\frac{\Delta t}{2} \frac{\partial f_{n+1}}{\partial x_{n+1}}\right)=$ anterior, obtém-se uma metodologia robusta capaz de = Qstimar os parâmetros do gerador síncrono sem a necessidade de desconectá-lo do sistema (Figura 1).

$\frac{\partial L}{\partial z_{n+1}}=2 g_{n+1} \frac{\partial g_{n+1}}{\partial z_{n+1}}+\lambda_{L(n+1)}\left(-\frac{\Delta t}{2}\right) \frac{\partial f_{n+1}}{\partial z_{n+1}}=0$,

$\frac{\partial L}{\partial \lambda_{L n+1}}=x_{n+1}-x_{n}-\frac{\Delta t}{2}\left(f_{n}+f_{n+1}\right)=0$.

Os sub-índices ' $n$ ' $e^{\text {' }} \mathrm{n}+1$ ' indicam o valor das variáveis $x, z$ e $u$ nos tempos discretos ' $n$ ' $\mathrm{e}$ ' $\mathrm{n}+1$ ', respectivamente. O método de Newton pode ser usado para resolver (10). A saída $y(t)$ pode ser calculada por simples substituição depois que $x(t)$ e $z(t)$ sejam estimados. As funções de sensibilidade podem ser determinadas derivando-se parcialmente (10) em relação a cada parâmetro.

O algoritmo da metodologia proposta para estimação de parâmetros de sistemas dinâmicos não lineares na forma de (7) é apresentado a seguir:

a. Selecione a estrutura do modelo e os parâmetros desconhecidos a serem estimados.

b. Escolha uma estimativa inicial de parâmetros $p^{(0)}=$ $p_{o}$ e faça $\mathrm{k}=0$.

c. Resolva o problema de minimização (8) e calcule as saídas do modelo $y_{(t)}$.

d. Compare as saídas do "Sistema Real" com as saídas do modelo de acordo com (3).

e. Pare se a equação algébrica $g\left(x_{n}, z_{n}, p^{(k)}, u\right)$ é igual a zero para cada "n" e o funcional $J\left(p^{(k)}\right)$ é inferior a um erro predefinido. Caso contrário continue.

f. Obtenha as equações de sensibilidade derivando-se (10) em relação a cada parâmetro $p_{i}$ e obtenha $\frac{\partial y}{\partial p}$.

g. Determine $\Gamma$ usando a equação $(6)$ e $\frac{\partial J(p)}{\partial p}$ usando a equação (4).

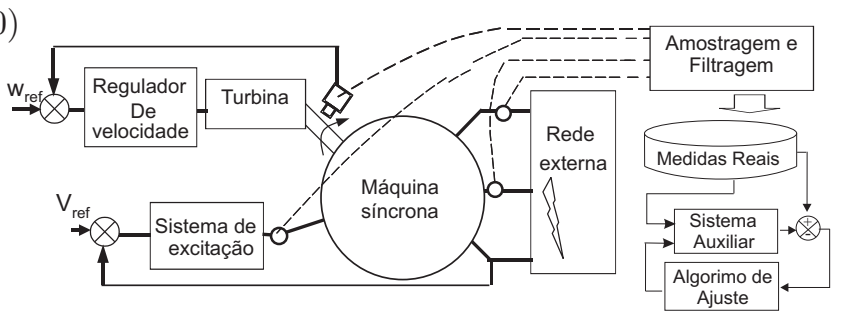

Figura 1: Esquema para estimação dos parâmetros a partir de medidas de perturbações.

Todas as variáveis dinâmicas que relacionam o gerador com o sistema são monitoradas. Mais especificamente monitoram-se as tensões e correntes trifásicas, a tensão de campo $^{2}$ e a velocidade de rotação do rotor. A potência mecânica $P_{m}$ é considerada como uma entrada constante para curtos períodos de tempo. Quando uma perturbação externa acontece no sistema de energia elétrica, estas medidas são armazenadas em uma base de dados para posterior processamento pelo algoritmo proposto de estimação de parâmetros.

O modelo de dois eixos para o gerador síncrono é dado por:

\footnotetext{
${ }^{2}$ Se a tensão de campo não estiver disponível (sistema de excitação sem escovas), pode-se modelar o sistema de excitação e estimar simultaneamente os parâmetros deste modelo e do gerador. Neste caso, a entrada do sistema de excitação é usada como entrada do modelo.
} 


$$
\begin{aligned}
\dot{\delta} & =\omega \\
\dot{\omega} & =\frac{\omega_{o}}{2 H}\left[P_{m}-P_{e}-\frac{D}{\omega_{o}} \omega\right], \\
P_{e} & =E_{q}^{\prime} I_{q}+E_{d}^{\prime} I_{d}+\left(x_{d}^{\prime}-x_{q}^{\prime}\right) I_{d} I_{q}, \\
\dot{E}_{q}^{\prime} & =\frac{1}{T_{d o}^{\prime}}\left[E_{f d}-E_{q}^{\prime}+\left(x_{d}-x_{d}^{\prime}\right) I_{d}\right], \\
\dot{E_{d}^{\prime}} & =\frac{-1}{T_{q o}^{\prime}}\left[E_{d}^{\prime}+\left(x_{q}-x_{q}^{\prime}\right) I_{q}\right], \\
I_{d} & =\frac{V_{t} \cos (\beta)-E_{q}^{\prime}}{x_{d}^{\prime}} \\
I_{q} & =\frac{V_{t} \operatorname{sen}(\beta)+E_{d}^{\prime}}{x_{q}^{\prime}} .
\end{aligned}
$$

Este modelo possui 4 variáveis de estado $\left(\delta, \omega, E_{q}^{\prime}, E_{d}^{\prime}\right)$ e 8 parâmetros $\left(x_{d}, x_{d}^{\prime}, T_{d o}^{\prime}, x_{q}, x_{q}^{\prime}, T_{q o}^{\prime}, H, D\right)$. A variável $\beta$ é o ângulo entre o eixo em quadratura e a tensão terminal $V_{t}$. Os estados $E_{q}^{\prime}$ e $E_{d}^{\prime}$ não podem ser medidos e $\delta(t)$ é difícil de ser medido em grandes geradores. Portanto, a metodologia precisará estimar também as condições iniciais destas variáveis $\left(E_{q o}^{\prime}, E_{d o}^{\prime}, \delta_{o}\right)$, as quais serão tratadas como parâmetros. Assim, o número de parâmetros a serem estimados aumenta para 11.

Além dos problemas numéricos usualmente encontrados para estimar um grande número de parâmetros simultaneamente, a aplicação da metodologia de sensibilidade de trajetória ao modelo (11)-(17) tem um problema intrínseco. O ângulo $\beta$ é o ângulo entre o 'eixo $-q$ ' e a tensão terminal, enquanto $\delta$ é o ângulo do 'eixo - q' em relação à referência síncrona. A medida da velocidade mecânica do rotor e a estimação de $\delta$ não são suficientes para estimar $\beta$ com precisão. Em outras palavras, $\beta$ não é observável a partir das medidas disponíveis. O uso de PMUs clássicos não é suficiente para obter esta medida, sendo necessário um medidor especial de ângulo de rotor APMU (Angle Phase Measurement Unit) como o apresentado em (Jin et al., 2007). Nesse caso, os parâmetros podem ser estimados usando a metodologia proposta em (Cari et al., 2006). Entretanto, o uso de APMUs ainda é muito limitado.

A metodologia de estimação de parâmetros proposta nesta artigo resolve este problema intrínseco do modelo e diminui o número de parâmetros a serem estimados simultaneamente. Para isto, duas ações serão tomadas:(i) o modelo será desacoplado para que a estimação dos parâmetros mecânicos e elétricos seja feita de forma independente e (ii) o ângulo $\beta$ será estimado usando uma equação algébrica adicional que relaciona o ângulo $\beta$ com as correntes elétricas.

\subsection{Estimação dos Parâmetros Mecânicos}

Para estimar os parâmetros mecânicos do gerador síncrono, considere a equação (12):

$$
\dot{\omega}=\frac{\omega_{o}}{2 H}\left[P_{m}-P_{e}-\frac{D}{\omega_{o}} \omega\right]
$$

As potências mecânica e elétrica $\left(P_{m}\right.$ e $P_{e}$ respectivamente) são consideradas como variáveis de entrada, enquanto que a velocidade do rotor $\omega$ é variável de saída. A potência mecânica $P_{m}$ é considerada constante e igual ao valor da potência elétrica $P_{e}$ antes da perturbação. A potência ativa $P_{e}$ é calculada a partir das tensões e correntes trifásicas medidas. Este modelo é completamente desacoplado das variáveis e parâmetros elétricos e contém uma variável de estado $\omega$ e dois parâmetros ' $H$ ' e ' $D$ ' (constante de inércia e coeficiente de amortecimento respectivamente).

Como a potência elétrica é uma medida de entrada, os efeitos de amortecimento devido ao enrolamento amortecedor são automaticamente considerados e não influenciam a estimação de ' $D$ '. Neste caso, ' $D$ ' representa apenas os efeitos de amortecimento mecânico.

As funções de sensibilidade são obtidas derivando-se (18) em relação aos dois parâmetros, isto é:

$$
\begin{aligned}
& \dot{\lambda}_{\omega}^{H}=\frac{-\omega_{o}}{2 H^{2}}\left(P_{m}-P_{e}-\frac{D}{\omega_{o}} \omega\right)+\frac{\omega_{o}}{2 H}\left(\frac{-D}{\omega_{o}} \lambda_{\omega}^{H}\right)(19) \\
& \dot{\lambda}_{\omega}^{D}=\frac{\omega_{o}}{2 H}\left(-\frac{\omega}{\omega_{o}}\right)+\frac{\omega_{o}}{2 H}\left(-\frac{D}{\omega_{o}} \lambda_{\omega}^{D}\right)
\end{aligned}
$$

onde $\lambda_{\omega}^{H}=\frac{\partial \omega}{\partial H}$ e $\lambda_{\omega}^{D}=\frac{\partial \omega}{\partial D}$ são as funções de sensibilidade da saída $\omega$ com relação aos parâmetros $H$ e $D$ respectivamente.

Utilizando a metodologia tradicional de sensibilidade de trajetória apresentada na seção 2 e as equações (18) e (19), os parâmetros mecânicos do gerador podem ser estimados.

\subsection{Estimação dos Parâmetros Elétricos}

Para estimar os parâmetros elétricos, utilizaremos a equação algébrica a seguir para estabelecer a relação entre o ângulo $\beta$ a ser estimado e a corrente $I$ medida:

$$
I^{2}-I_{d}^{2}-I_{q}^{2}=0 .
$$

As potências elétricas ativa e reativa serão as saídas e a corrente elétrica $I$, a tensão terminal do gerador $V_{T}$ e a 
tensão de campo $E_{f d}$ serão as entradas. O modelo usado para estimar os parâmetros elétricos é portanto constituído pelas equações diferenciais (14)-(15), pela equação algébrica (20), as saídas $P_{e}$ e $Q_{e}$ e as variáveis intermediárias $I_{d}$ e $I_{q}$ (equações (16) e (17) respectivamente), isto é:

$$
\begin{aligned}
\dot{E}_{q}^{\prime} & =\frac{1}{T_{d o}^{\prime}}\left[E_{f d}-E_{q}^{\prime}+\left(x_{d}-x_{d}^{\prime}\right) I_{d}\right] \\
\dot{E}_{d}^{\prime} & =\frac{-1}{T_{q o}^{\prime}}\left[E_{d}^{\prime}+\left(x_{q}-x_{q}^{\prime}\right) I_{q}\right] \\
0 & =I^{2}-I_{d}^{2}-I_{q}^{2} \\
P_{e} & =E_{d}^{\prime} I_{d}+E_{q}^{\prime} I_{q}+\left(x_{d}^{\prime}-x_{q}^{\prime}\right) I_{d} I_{q} \\
Q_{e} & =E_{d}^{\prime} I_{q}-E_{q}^{\prime} I_{d}-x_{q}^{\prime} I_{q}^{2}-x_{d}^{\prime} I_{d}^{2} \\
I_{d} & =\frac{V_{t} \cos (\beta)-E_{q}^{\prime}}{x_{d}^{\prime}} \\
I_{q} & =\frac{V_{t} \operatorname{sen}(\beta)+E_{d}^{\prime}}{x_{q}^{\prime}}
\end{aligned}
$$

Este modelo contém oito parâmetros $p=\left(x_{d}, x_{d}^{\prime}, T_{d o}^{\prime}, x_{q}, x_{q}^{\prime}, T_{q o}^{\prime}, E_{q o}^{\prime}, E_{d o}^{\prime}\right)^{T}$, duas variáveis de estado $x=\left(E_{q}^{\prime}(t), E_{d}^{\prime}(t)\right)$ e uma variável algébrica $z=(\beta(t))$. O vetor de entrada é $u=\left(E_{f d}(t), I(t), V(t)\right)$ e o vetor de saída é $y=\left(P_{e}(t), Q_{e}(t)\right)$.

Com o objetivo de contornar os problemas de não solvabilidade da equação algébrica (23), as equações do modelo (21)-(23) serão substituídas por um problema de minimização restrito. Mais precisamente, ao invés de resolver (21)-(23), o quadrado da função $g$ é minimizado, restrito à equação diferencial, isto é:

$$
\begin{aligned}
& \min _{(x, z)} \quad g^{2}\left(E_{q}^{\prime}, E_{d}^{\prime}, \beta, p, I, V_{t}, E_{f d}\right) \\
& \text { s.a }\left\{\begin{array}{rrr}
\dot{E}_{q}^{\prime} & = & \frac{1}{T_{d o}^{\prime}}\left[E_{f d}-E_{q}^{\prime}+\left(x_{d}-x_{d}^{\prime}\right) I_{d}\right] \\
\dot{E}_{d}^{\prime} & = & \frac{-1}{T_{q o}^{\prime}}\left[E_{d}^{\prime}+\left(x_{q}-x_{q}^{\prime}\right) I_{q}\right]
\end{array}\right. \\
& \text { onde } g(\overbrace{E_{q}^{\prime}, E_{d}^{\prime}}^{x}, \overbrace{\beta}^{z}, p, \overbrace{I, V_{t}, E_{f d}}^{u})=I^{2}-I_{d}^{2}-I_{q}^{2} \text {. }
\end{aligned}
$$

O problema de estimação de parâmetros de geradores síncronos pode agora ser resolvido por intermédio da abordagem de minimização proposta na Seção 3 para estimação de parâmetros de sistemas dinâmicos modelados por um conjunto de equações algébrico-diferenciais. $\mathrm{O}$ equacionamento detalhado desta nova metodologia de estimação de parâmetros de geradores pode ser encontrado em (Cari, 2009).

\section{TESTES}

Nesta seção, a metodologia de estimação de parâmetros de geradores síncronos, proposta na seção anterior, é testada em um sistema de energia de pequeno porte cuja topologia é ilustrada na Figura 2. O gerador síncrono tem um sistema de excitação tipo ST1A (IEEE, 1992) com os parâmetros dados em pu.: $K_{A}=200, T_{A}=0.025$, $E f_{\min }=-6.4$ e $E f_{\max }=7$.

Diversos testes foram realizados com dados de perturbações oriundos de faltas monofásicas, bifásicas, bifásicas a terra, trifásicas e abertura de uma linha de transmissão. Devido a limitação de espaço, apenas os resultados de estimação obtidos com dados de faltas monofásicas e faltas trifásicas serão apresentados neste artigo. Apesar disto, os resultados obtidos com os outros tipos de perturbações também foram satisfatórios. Dois testes foram realizados:

Teste 1 Um curto-circuito fase-terra ocorre no instante $t=0$ em uma das fases da linha de transmissão $L_{2}$ (Figura 2). O curto é isolado pelo acionamento dos disjuntores da linha em falta em $t=0,15 \mathrm{~s}$.

Teste 2 Um curto-circuito trifásico ocorre na linha de transmissão $L_{2}$ no instante $t=0$. O curto é isolado pelo acionamento dos disjuntores da linha em $t=$ $0,07 \mathrm{~s}$.

As medidas de correntes e tensões trifásicas, tensão de campo e velocidade do gerador foram obtidas por simulação usando o software Matlab. A partir destas medidas, calculam-se as componentes de sequência positiva das tensões e correntes trifásicas e obtêm-se os fasores de tensão e corrente, assim como a potência ativa e reativa nos terminais do gerador.

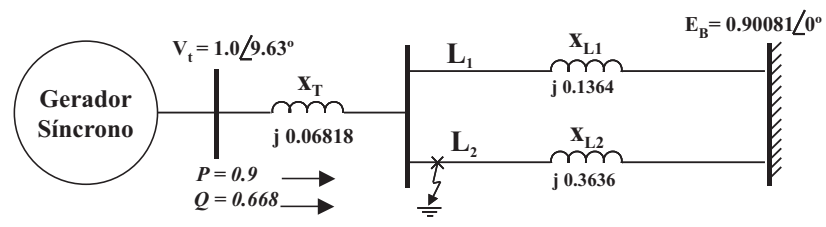

Figura 2: Sistema de potência usado para os testes. As medidas são coletadas para serem usadas pela metodologia.

A frequência de amostragem foi de $1 \mathrm{kHz}$. A janela de tempo amostral foi de 2 segundos para estimar os parâmetros elétricos e 0,5 segundos para estimar os parâmetros mecânicos. 


\subsection{Parâmetros Mecânicos}

Nesta seção, apresentam-se os resultados obtidos com a aplicação da metodologia de sensibilidade de trajetória, apresentada na seção 2, para estimar os parâmetros $H$ e $D$ do gerador a partir das medidas obtidas no teste 1 . Verificou-se que os parâmetros mecânicos podem ser facilmente estimados independentemente dos parâmetros elétricos, usando o modelo (18) e a metodologia tradicional de sensibilidade de trajetória.

Os valores iniciais dos parâmetros mecânicos foram perturbados em até $\pm 90 \%$ de seus valores nominais. Os parâmetros convergiram a seus valores verdadeiros após 7 iterações conforme resultados da tabela 1. Nas figu-

Tabela 1: Estimação dos parâmetros mecânicos.

\begin{tabular}{|c|c|c|c|c|c|}
\hline$P$. & $\begin{array}{l}\text { Val. } \\
\text { Ini. }\end{array}$ & & $\begin{array}{l}\text { Val. } \\
\text { Fin. }\end{array}$ & $\begin{array}{c}\text { Val. } \\
\text { Verd. }\end{array}$ & Erro(\%) \\
\hline$H$ & 6.65 & $+90 \%$ & 3.500 & 3.5 & $0.0(\%)$ \\
\hline$D$ & 0.09 & $-90 \%$ & 0.899 & 0.9 & $-0.11(\%)$ \\
\hline
\end{tabular}

ras (3)-(4) comparam-se as saídas do sistema real (linha sólida) e do modelo (linha tracejada) antes e após a convergência do processo iterativo de estimação de parâmetros. Observa-se, após convergência, um perfeito casamento entre as saídas.

A estimação dos parâmetros mecânicos foi realizada sem problemas e com relativa facilidade em todos os testes realizados, indicando que a metodologia tradicional de sensibilidade de trajetória é robusta o suficiente para a estimação destes parâmetros.

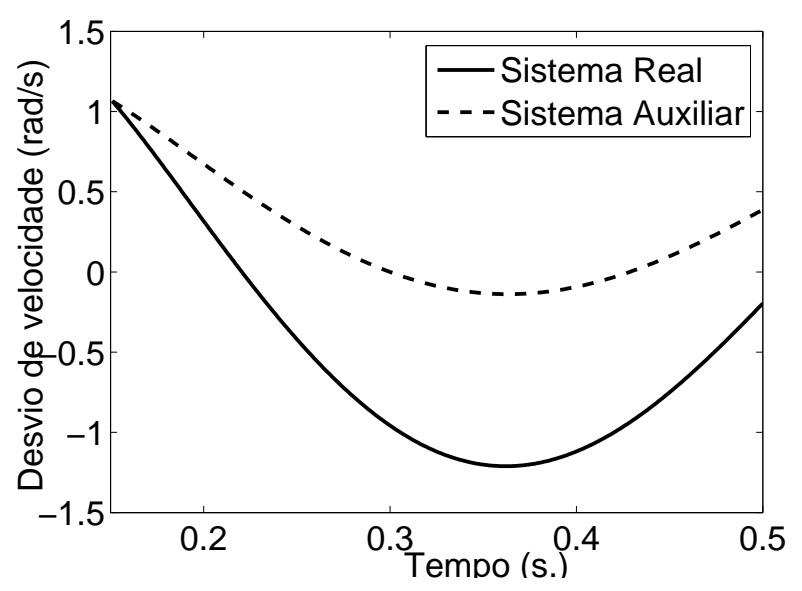

Figura 3: Desvio de velocidade no começo.

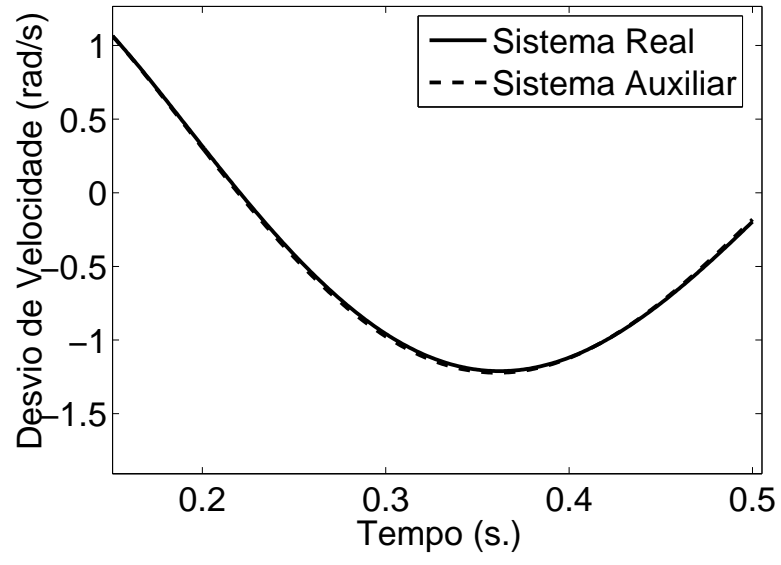

Figura 4: Desvio de velocidade no final.

\subsection{Parâmetros Elétricos}

Nesta seção, apresentam-se os resultados dos testes obtidos com o emprego da metodologia de estimação de parâmetros elétricos de geradores síncronos proposta na seção 3 .

A experiência dos autores no emprego da metodologia clássica de sensibilidade de trajetória para a estimação de parâmetros do gerador indica que a convergência do processo iterativo de ajuste de parâmetros só é garantida se os valores iniciais dos parâmetros estiverem muito próximos dos valores verdadeiros, especialmente os parâmetros $\beta_{o}, E_{q o}^{\prime}$ e $E_{d o}^{\prime}$. Como os valores destas condições iniciais dependem do ponto de operação do sistema, é difícil obter boas estimativas iniciais destes parâmetros. Neste artigo consideramos os seguintes valores iniciais gerais para estes parâmetros:

$\beta_{o}=\tan ^{-1}\left(\frac{P_{e}}{Q_{e}+V_{T}^{2} / x_{d}}\right)$ (aproximação para máquinas de pólos lisos em regime permanente) e $E_{q o}^{\prime}=V_{t o}, E_{d o}^{\prime}=0$ (máquina em vazio).

Quando todos os parâmetros tentam ser estimados simultaneamente, a abordagem tradicional de sensibilidade de trajetória apresentada na Seção 2 só consegue estimar os parâmetros se os valores iniciais dos parâmetros possuírem erros de no máximo $\pm 2 \%$ em relação aos valores verdadeiros (região de convergência $\pm 2 \%$ ). Para erros maiores nos parâmetros, problemas de não solvabilidade da equação algébrica são observados e os parâmetros não podem ser estimados por esta abordagem.

Utilizando a metodologia proposta (abordagem de minimização da Seção 3) para estimar os parâmetros elétricos do gerador (equação 28), a região de convergência 
aumenta significativamente, permitindo a correta estimação dos parâmetros a partir de valores iniciais dos parâmetros com erros entre $-20 \%$ a $+25 \%$ com os dados do teste 1 (falta monofásica) e entre $-35 \%$ e $+67 \%$ com os dados do teste 2 (falta triásica). Neste caso, os parâmetros foram previamente classificados em parâmetros com baixa e alta sensibilidade segundo decomposição QR apresentada na Seção 2.1 e estimados em duas fases (Tabela 2).

Tabela 2: Valores singulares da matriz de sensibilidade e parâmetros classificados segundo sua influência na saída.

\begin{tabular}{|c|c|c|c|c|c|c|c|}
\hline$x_{d}^{\prime}$ & $E_{q o}^{\prime}$ & $E_{d o}^{\prime}$ & $x_{d}$ & $x_{q}^{\prime}$ & $T_{q o}^{\prime}$ & $x_{q}$ & $T_{d o}^{\prime}$ \\
\hline 3.23 & 0.65 & 0.32 & 0.042 & 0.029 & 0.009 & 0.002 & 0.001 \\
\hline
\end{tabular}

Segundo esta decomposição, os parâmetros $T_{d o}^{\prime}, x_{q}$ e $T_{q o}^{\prime}$ tem pequena sensibilidade e menos influência na saída do modelo, e portanto, foram mantidos fixos nas primeiras iterações.

Os resultados da estimação com os dados do teste 1 (falta monofásica) são mostrados na Tabela 3. A coluna "Fase 1" apresenta o resultado intermediário da estimação quando os parâmetros com baixa sensibilidade foram mantidos fixos. A coluna "Fase 2" apresenta o resultado final da metodologia proposta de estimação de parâmetros. O tempo de processamento, até a convergência da segunda fase, é menor do que 2 minutos em um computador com processador Pentium, DualCore de $2 \mathrm{GHz}$.

Tabela 3: Estimação dos parâmetros elétricos com dados do teste 1 (falta monofásica).

\begin{tabular}{|c|c|c|c|c|c|c|}
\hline $\mathrm{p}$ & $\begin{array}{c}\text { Valor } \\
\text { inicial }\end{array}$ & Desvio & $\begin{array}{c}\text { Fase } \\
1\end{array}$ & $\begin{array}{c}\text { Fase } \\
2\end{array}$ & $\begin{array}{c}\text { Valor } \\
\text { Nom. }\end{array}$ & $\begin{array}{c}\text { Erro } \\
(\%)\end{array}$ \\
\hline$x_{d}$ & 2.26 & $+25 \%$ & 1.70 & 1.81 & 1.81 & 0.0 \\
\hline$x_{d}^{\prime}$ & 0.38 & $+25 \%$ & 0.25 & 0.30 & 0.30 & 0.0 \\
\hline$T_{d o}^{\prime}$ & 10.0 & $+25 \%$ & 10.0 & 7.98 & 8.00 & -0.2 \\
\hline$x_{q}$ & 2.20 & $+25 \%$ & 2.20 & 1.76 & 1.76 & 0.0 \\
\hline$x_{q}^{\prime}$ & 0.81 & $+25 \%$ & 0.34 & 0.65 & 0.65 & 0.0 \\
\hline$T_{q o}^{\prime}$ & 1.25 & $+25 \%$ & 1.25 & 0.996 & 1.00 & -0.4 \\
\hline
\end{tabular}

É importante observar que, devido a impossibilidade de medi-las, as condições iniciais $E_{q o}^{\prime}$ e $E_{d o}^{\prime}$ das variáveis de estado $E_{q}^{\prime}$ e $E_{d}^{\prime}$ são tratadas como parâmetros pela metodologia de estimação. O valor inicial utilizado para $E_{q o}^{\prime}$ foi a tensão terminal no início da amostragem ( $\left.V_{t o}=1.03\right)$ e o valor inicial de $E_{d o}^{\prime}$ foi nulo. Embora estes valores iniciais não estejam muito próximos dos valores reais $\left(E_{q o}^{\prime}=1,20\right.$ e $E_{d o}^{\prime}=-0,328$ para a falta monofásica), a metodologia proposta estimou os parâmetros com sucesso ao passo que a metodologia clássica de sensibilidade de trajetória não convergiu. A metodologia clássica requer estimativas muito boas destes valores ao passo que a metodologia proposta obteve êxito com esta abordagem geral e prática de escolha de condições iniciais para estas variáveis.

Os resultados da estimação com os dados do teste 2 (falta trifásica) são mostrados na tabela 4. As Figuras 5-6 mostram os valores da função algébrica $g^{2}(x, z, p, u)$ no começo e no final do processo de estimação com medidas do teste 2 . No começo do processo de estimação, a equação $g=0$ não tem solução em alguns intervalos, comprovando que a abordagem de sensibilidade de trajetória tradicional não pode ser aplicada e justificando a necessidade de aplicação da metodologia proposta. Como esperado, a função algébrica se anula após convergência.

Tabela 4: Estimação dos parâmetros elétricos para o ensaio 2 (falta trifásica).

\begin{tabular}{|c|c|c|c|c|c|c|}
\hline $\mathrm{p}$ & $\begin{array}{c}\text { Valor } \\
\text { inicial }\end{array}$ & Desvio & $\begin{array}{c}\text { Fase } \\
1\end{array}$ & $\begin{array}{c}\text { Fase } \\
2\end{array}$ & $\begin{array}{c}\text { Valor } \\
\text { Nom. }\end{array}$ & $\begin{array}{c}\text { Erro } \\
(\%)\end{array}$ \\
\hline$x_{d}$ & 1.21 & $-33 \%$ & 1.83 & 1.81 & 1.81 & 0.0 \\
\hline$x_{d}^{\prime}$ & 0.20 & $-33 \%$ & 0.35 & 0.30 & 0.30 & 0.0 \\
\hline$T_{d o}^{\prime}$ & 5.36 & $-33 \%$ & 5.36 & 7.99 & 8.00 & -0.1 \\
\hline$x_{q}$ & 1.18 & $-33 \%$ & 1.18 & 1.76 & 1.76 & 0.0 \\
\hline$x_{q}^{\prime}$ & 0.44 & $-33 \%$ & 1.15 & 0.65 & 0.65 & 0.0 \\
\hline$T_{q o}^{\prime}$ & 0.67 & $-33 \%$ & 0.67 & 0.996 & 1.00 & -0.4 \\
\hline
\end{tabular}

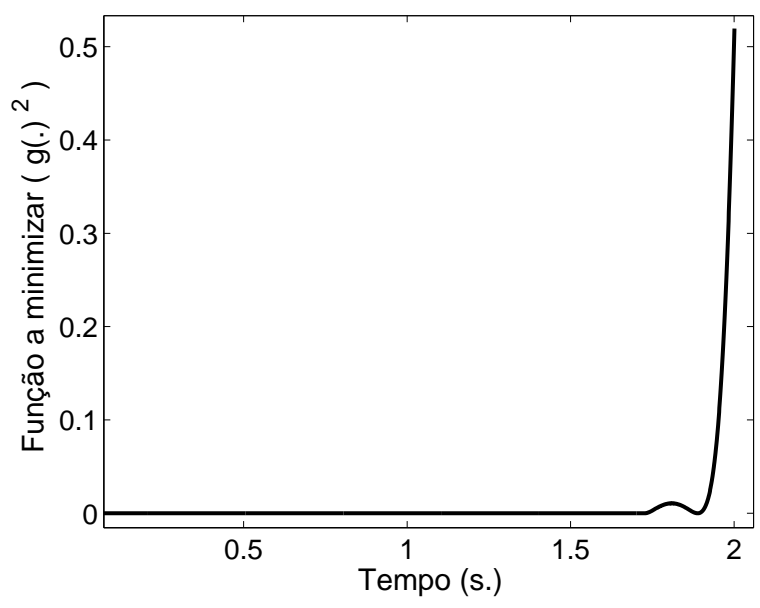

Figura 5: Função $g$ no começo das iterações. 


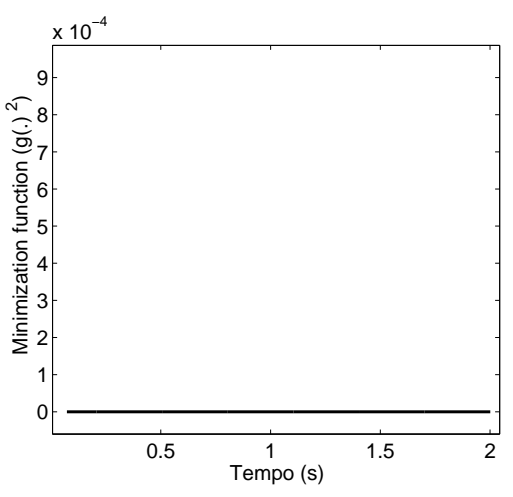

Figura 6: Função $g$ no final das iterações.

\subsection{Influência do Ruído nas Medidas}

A metodologia proposta de estimação de parâmetros de geradores síncronos também foi testada considerando-se ruído nas medidas com média zero e desvio padrão de 1\%. Para os parâmetros mecânicos, o ruído teve pouca influência na precisão dos parâmetros estimados. A região de convergência também não foi comprometida pelo ruído (vide Tabela 5).

Tabela 5: Influência do ruído na estimação dos parâmetros mecânicos.

\begin{tabular}{|c|c|c|c|c|c|}
\hline$p$ & $\begin{array}{c}\text { Valor } \\
\text { inicial }\end{array}$ & Desvio & $\begin{array}{c}\text { Valor } \\
\text { final }\end{array}$ & $\begin{array}{c}\text { Valor } \\
\text { nominal }\end{array}$ & $\begin{array}{c}\text { Erro } \\
(\%)\end{array}$ \\
\hline$H$ & 6.65 & $+90 \%$ & 3.504 & 3.50 & $0.11(\%)$ \\
\hline$D$ & 0.09 & $-90 \%$ & 0.864 & 0.9 & $-4.0(\%)$ \\
\hline
\end{tabular}

No caso dos parâmetros elétricos, o maior erro para o teste 1 foi no parâmetro $x_{q}^{\prime}$ que convergiu com um erro de $+6 \%$. A região de convergência entretanto não foi comprometida.

Para o teste 2, o maior erro foi no parâmetro $E_{d o}^{\prime}$ que convergiu com um erro de $+0.9 \%$. A região de convergência também não foi comprometida. Na figura 7 mostra-se o casamento da potência elétrica $\left(P_{e}\right)$ medida e calculada no final do processo de estimação com ruído nas medidas. A figura 8 é uma ampliação da figura 7 .

Observou-se que a metodologia proposta via abordagem de minimização é robusta a ruídos e a erros nos valores iniciais dos parâmetros e portanto viabiliza a aplicação da técnica de sensibilidade de trajetória no problema de estimação de parâmetros elétricos de geradores síncronos.

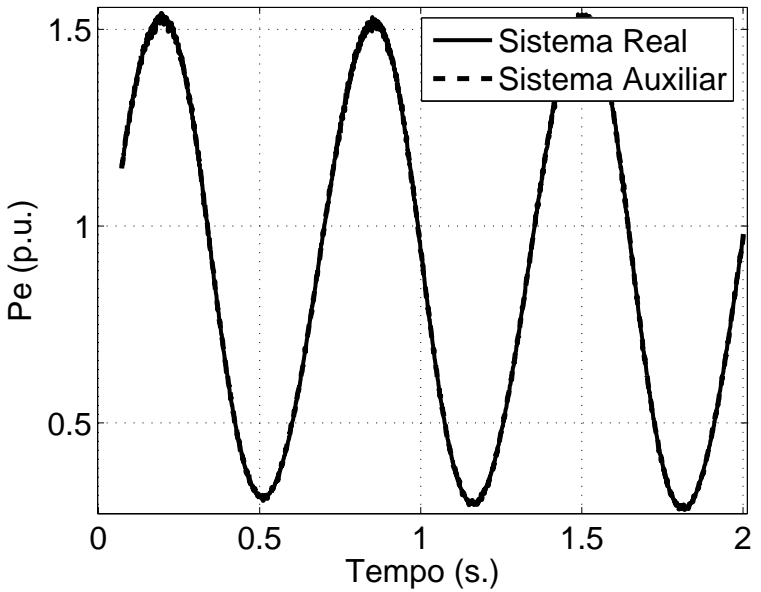

Figura 7: Convergência de $P_{e}$ com ruído.

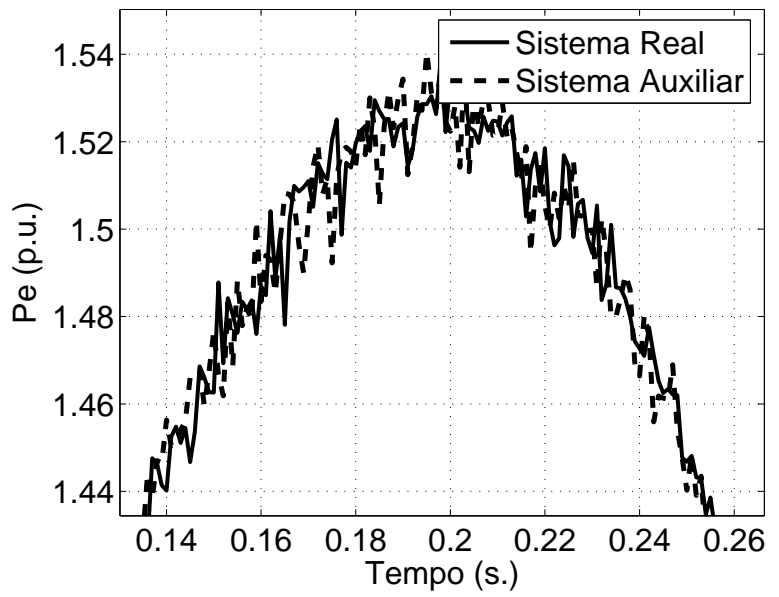

Figura 8: Ampliação da convergência de $P_{e}$

\subsection{Influência das Condições Iniciais}

A robustez da metodologia proposta foi testada, estimando-se os parâmetros do gerador a partir de diferentes casos (valores iniciais dos parâmetros). Os resultados destes testes são apresentados a seguir:

\section{Estimação de parâmetros mecânicos}

As medidas foram obtidas usando o teste 1 (falta monofásica), com ruído de média zero e desvio padrão de 0,5\%. Conforme tabela 6 , todos os parâmetros convergiram em menos de 8 iterações (Iter) com erros inferiores a $0,3 \%$. O tempo máximo de processamento foi inferior a $4 \mathrm{seg}$.

\section{Estimação de parâmetros elétricos}


Tabela 6: Estimação dos parâmetros mecânicos.

\begin{tabular}{|c|c|c|c|c|c|c|}
\hline Caso & & $\begin{array}{c}\text { Val. } \\
\text { Ini. }\end{array}$ & $\begin{array}{c}\text { Val. } \\
\text { Fin. }\end{array}$ & $\begin{array}{c}\text { Val. } \\
\text { Verd. }\end{array}$ & $\begin{array}{c}\text { Erro } \\
(\%)\end{array}$ & Iter. \\
\hline \multirow{3}{*}{1} & $H$ & 0.1 & 3.5019 & 3.50 & 0.054 & \\
\hline & $D$ & 0.1 & 0.8945 & 0.90 & -0.297 & 8 \\
\hline & $H$ & 6.0 & 3.5016 & 3.50 & 0.052 & \\
\hline \multirow{3}{*}{3} & $D$ & 0.0 & 0.8972 & 0.90 & -0.266 & 4 \\
\hline & $D$ & 2.0 & 3.5019 & 3.50 & 0.053 & \\
4 & $H$ & 0.2 & 0.8947 & 0.90 & -0.279 & 4 \\
\hline & $D$ & 0.5 & 3.5016 & 3.50 & 0.040 & \\
\end{tabular}

As medidas para estimar os parâmetros elétricos foram obtidas do teste 2 (falta trifásica) com ruído de média zero e desvio padrão $0,5 \%$. A escolha dos valores iniciais dos parâmetros é arbitrária, desde que esteja dentro da região de convergência, definida na Seção 5.2 (-35\% e $+67 \%$ do valor nominais) e sejam mantidas as restrições físicas de parâmetros de máquinas elétricas: $x_{d}>x_{q}>$ $x_{q}^{\prime}>x_{d}^{\prime}$ e $T_{d o}^{\prime}>T_{q o}^{\prime}$. Foram testadas várias situações, porém, apenas 3 casos são apresentados.

Tabela 7: Estimação dos parâmetros elétricos (caso 1).

\begin{tabular}{|c|c|c|c|c|c|}
\hline p. & $\begin{array}{c}\text { Valor } \\
\text { inicial }\end{array}$ & $\begin{array}{c}\text { Fase } \\
1\end{array}$ & $\begin{array}{c}\text { Fase } \\
2\end{array}$ & $\begin{array}{c}\text { Valor } \\
\text { Nom. }\end{array}$ & $\begin{array}{c}\text { Erro } \\
(\%)\end{array}$ \\
\hline$x_{d}$ & 2.20 & 1.73 & 1.809 & 1.81 & -0.05 \\
\hline$x_{d}^{\prime}$ & 0.50 & 0.26 & 0.300 & 0.30 & 0.16 \\
\hline$T_{d o}^{\prime}$ & 10.0 & 10.0 & 7.980 & 8.00 & -0.25 \\
\hline$x_{q}$ & 2.00 & 2.00 & 1.750 & 1.76 & -0.55 \\
\hline$x_{q}^{\prime}$ & 0.80 & 0.45 & 0.653 & 0.65 & 0.40 \\
\hline$T_{q o}^{\prime}$ & 1.40 & 1.40 & 0.986 & 1.00 & -1.36 \\
\hline
\end{tabular}

Tabela 8: Estimação dos parâmetros elétricos (caso 2).

\begin{tabular}{|c|c|c|c|c|c|}
\hline p. & $\begin{array}{c}\text { Valor } \\
\text { inicial }\end{array}$ & $\begin{array}{c}\text { Fase } \\
1\end{array}$ & $\begin{array}{c}\text { Fase } \\
2\end{array}$ & $\begin{array}{c}\text { Valor } \\
\text { Nom. }\end{array}$ & $\begin{array}{c}\text { Erro } \\
(\%)\end{array}$ \\
\hline$x_{d}$ & 1.70 & 1.87 & 1.809 & 1.81 & -0.05 \\
\hline$x_{d}^{\prime}$ & 0.40 & 0.29 & 0.301 & 0.30 & 0.16 \\
\hline$T_{d o}^{\prime}$ & 7.0 & 7.0 & 7.980 & 8.00 & -0.24 \\
\hline$x_{q}$ & 1.50 & 1.502 & 1.750 & 1.76 & -0.55 \\
\hline$x_{q}^{\prime}$ & 0.80 & 0.625 & 0.652 & 0.65 & 0.40 \\
\hline$T_{q o}^{\prime}$ & 1.40 & 1.40 & 0.986 & 1.00 & -1.36 \\
\hline
\end{tabular}

Os parâmetros convergiram em todos os casos em menos de 12 iterações com erros inferiores a 1,4\%, com um tempo de processamento inferior a 2 minutos.

\section{CONCLUSÕES}

Neste trabalho, uma nova metodologia para estimar parâmetros de geradores síncronos (modelo de dois-eixos)
Tabela 9: Estimação dos parâmetros elétricos (caso 3).

\begin{tabular}{|c|c|c|c|c|c|}
\hline p. & $\begin{array}{c}\text { Valor } \\
\text { inicial }\end{array}$ & $\begin{array}{c}\text { Fase } \\
1\end{array}$ & $\begin{array}{c}\text { Fase } \\
2\end{array}$ & $\begin{array}{c}\text { Valor } \\
\text { Nom. }\end{array}$ & $\begin{array}{c}\text { Erro } \\
(\%)\end{array}$ \\
\hline$x_{d}$ & 2.40 & 1.79 & 1.81 & 1.81 & -0.05 \\
\hline$x_{d}^{\prime}$ & 0.40 & 0.35 & 0.301 & 0.30 & 0.16 \\
\hline$T_{d o}^{\prime}$ & 6.40 & 6.40 & 7.980 & 8.00 & -0.24 \\
\hline$x_{q}$ & 2.30 & 2.30 & 1.750 & 1.76 & -0.55 \\
\hline$x_{q}^{\prime}$ & 0.80 & 0.92 & 0.653 & 0.65 & 0.40 \\
\hline$T_{q o}^{\prime}$ & 0.80 & 0.80 & 0.986 & 1.00 & -1.36 \\
\hline
\end{tabular}

que combina técnicas de análise de sensibilidade de trajetória com uma nova abordagem de minimização foi proposta. A metodologia proposta satisfaz diversos requisitos práticos desejáveis para a solução do problema de estimação de parâmetros de geradores síncronos. A metodologia é robusta em relação a valores iniciais dos parâmetros, garantindo uma região de convergência grande. Esta robustez foi conseguida como resultado da nova abordagem de minimização, proposta neste artigo, e da escolha adequada de variáveis de entrada e saída. Os parâmetros foram estimados a partir de medidas de fácil obtenção e a estimativa dos parâmetros elétricos e mecânicos foi realizada de uma forma desacoplada. Embora a abordagem de minimização tenha sido utilizada neste trabalho para estimação de parâmetros de geradores síncronos, ela é geral e pode ser empregada para a estimação de parâmetros de sistemas dinâmicos não-lineares gerais modelados por conjunto de equações algébrico-diferenciais. Além disso, verificou-se que o ruído nas medidas não tem influência significativa na metodologia proposta.

\section{AGRADECIMENTOS}

Os autores agradecem a FAPESP (Fundação de Amparo a Pesquisa do Estado de Sao Paulo) e CNPq pelo apoio financeiro a esta pesquisa.

\section{REFERÊNCIAS}

Bazaraa, M. S. (1979). Nonlinear Programming, John Wiley and Sons, Inc.

Benchluch, S. and Chow, J. H. (1993). A trajectory sensitivity method for the identification of nonlinear excitation system models, IEEE Transaction on Energy Conversion 8: 159-164.

Burth, M., Verghese, G. C. and Velez, R. M. (1999). Subset selection for improved parameter estimation in on-line identification of a synchronous ge- 
nerator, IEEE Transaction on Power and Systems 14(1): 218-225.

Cari, E. P. T. (2009). Metodologia de estimação de parâmetros de sistemas dinâmicos não-lineares com aplicação em geradores sincronos, Tese de doutorado, Universidade de São Paulo, Escola de engenharia de São Carlos, http://www.teses.usp.br/teses/disponiveis/ 18/18154/tde-06052009-101122/pt-br.php.

Cari, E. P. T., Alberto, L. F. C. and Bretas, N. (2006). A methodology for parameter estimation of synchronous generators based on trajectory sensitivity and synchronization technique, Power Engineering Society General Meeting .

Hiskens, I. A. (2000). Trajectory sensitivity analysis of hybrid systems, IEEE Transactions on Circuits and Systems 47(2): 204-220.

Hiskens, I. A. and Koeman (1998). Parameter estimation from power system disturbance measurements, International Conference on Energy Management and Power Delivery 2: 667-672.

IEEE (1992). Recommended practice for excitation system models for power system stability studies, $T e$ chnical report, Std 421.5-1992, Power Engineering Society.

IEEE (2002). Guide for synchronous generator modeling practices and applications in power system stability analyses, Technical report, Std 1110-2002, Power Engineering Society.

Jin, Y. Q., Qin, C., Wu, F., Han, J. D., Xu, Q., Yan, D. J. and Ju, P. (2007). The power angle and phase measurement units based wide area measurement system and its application, IREP Symposium Bulk Power System Dynamics and Control.

Karrari, M. and Malik, O. P. (2004). Identification of physical parameter of a synchronous generator from online measurements, IEEE Transactions on Energy Conversion 19: 1-9.

Luenberger, D. G. (1972). Introduction to Linear and Nonlinear Programming, Addison Wesley.

Sanchez, G. J., Bridenbaugh, C. J., Bowler, C. and Edmonds, J. (1988). Trajectory sensitivity based identification of synchronous generator and excitation system parameters, IEEE Transaction on Power System 3: 1814-1822.
Vermeulen, H., Strauss, J. M. and J.M., S. (2002). Online estimation of synchronous generator parameters using prbs perturbations, IEEE Transactions on Power System 17: 694-700. 